\title{
The effect of personality on reasoning
}

Ascensión Fumero $^{1}$, Carlos Santamaría ${ }^{1}$ and Philip Johnson-Laird ${ }^{2}$

${ }^{1}$ Departamento de Personalidad, Evaluación y Tratamientos Psicológicos, Universidad de la Laguna, Campus de Guajara, 38205 Tenerife, Spain

${ }_{2}^{2}$ Psychology Department Princeton University Princeton, NJ 0854, US

The ability to reason depends on intelligence ${ }^{3}$, but preferences for different sorts of reasoning may depend on an individual's personality. The possibility is unlikely if reasoning is akin to a formal proof in logic ${ }^{4,5}$. But, such effects are predicted from the theory that reasoning is a semantic process of envisaging possibilities consistent with the meanings of premises and knowledge'. According to the standard 'big five' model of personality ${ }^{7}$, individuals differ in their openness to experience and in their conscientiousness. Certain inferential problems are ambiguous, e.g., if a pilot falls from a plane without a parachute then the pilot dies; this pilot didn't die; how come? A deductive response is: the pilot didn't fall from a plane without a parachute; an inductive response is: the plane was on the ground. Highly conscientious individuals who are also less open to experience should focus on the possibilities consistent with the premises, and tend to make a deduction, whereas those who are open to experience and not very conscientious should go beyond these possibilities, and tend to make an induction. Here we show that these biases occurred both for stable personality traits and for the same attitudes induced experimentally, either from false feedback about performance in a personality test or from participants' recall of autobiographical episodes illustrating the required attitudes. Researchers have often assumed that reasoning is a universal akin to the ability to use natural language ${ }^{4}$. They have also assumed that personality traits are stable with constant effects on cognition and behaviour ${ }^{8,9}$. Our results imply that neither assumption is invariably correct. They also corroborate the semantic 
view of human reasoning, and may lead to the discovery of other effects of personality traits, such as extraversion and neuroticism, on reasoning.

Not everyone thinks in the same way, and personality may influence the way people reason. According to the standard model of personality all individuals can be located in a five-dimensional space depending on the degree to which they have each of five major traits: openness to experience, conscientiousness, extraversion, agreeableness, and neuroticism. If reasoning is a formal process akin to formulating a proof in logic then, as theorists propose, a single deterministic theory should apply to everyone ${ }^{5}$. But, if reasoning depends instead on a semantic process in which individuals envisage possibilities consistent with the meanings of premises and their knowledge ${ }^{6}$, then personality should yield a preference between the two major sorts of reasoning: deduction vs. induction. In a valid deduction, the conclusion must hold in all the possibilities consistent with the premises, whereas in an induction, the conclusion goes beyond these possibilities to yield a plausible hypothesis or explanation. It follows that those who are not open to experience but highly conscientious should focus on the possibilities consistent with the premises, and therefore tend to make deductions, whereas those who are open to experience and not highly conscientious should go beyond the possibilities, and therefore tend to make inductions. Our research has corroborated this prediction.

Our experiments used problems that could be solved by either a deduction or an induction, e.g.: 'If you follow this diet then you lose weight. Ann did not lose weight. Why not?' A valid deduction underlies the answer: 'Ann didn't follow this diet,' but an induction underlies the answer: 'Ann had a metabolic problem'. Our first experiment tested 104 students from the University of La Laguna who completed the NEO-PI-R personality test on line. Two weeks later, they carried out ten reasoning problems, which each had the same form as the previous example but different contents. No 
relation between the two parts of the experiment was explicit to them, and two different experimenters recruited them. We confirmed a significant bimodal distribution $(\mathrm{n}=$ 104, Chi-square $=23.981 ; \mathrm{df} .=10 ; \mathrm{p}<0.008$; two-tailed) in the numbers of spontaneous inductions that participants tended to make given ten such problems (Fig. 1): those who were higher in conscientiousness than openness tended to make deductions (only 19\% inductions, see left-side of Fig.), whereas those who were higher in openness than conscientiousness tended to make inductions (81\%, see right-side of Fig; MannWhitney $U=56.5 ; z=2.16 ; p<0.02$; one-tailed). Previous research on personality and intelligence has shown that open individuals tend to be more intelligent than average ${ }^{10}$, but we found no reliable relation between our participants' intelligence or academic achievement and their personality traits or tendency to make inductions. PAU scores (university admission marks based on school grades and an examination) for 43 participants correlated with the proportion of inductions with $r=-0.264$. PAU scores correlated with the five traits as follows: Neuroticism, $r=0.104$, Extraversion $r=-0.178$, Openness $r=0.108$, Agreeableness $r=-0.079$, Conscientiousness $r=0.026$. Similarly, the correlations between Thurstone's Primary Mental Abilities (PMA) reasoning subscale ${ }^{11}$ for 24 participants and number of inductions was $r=-0.007$. It correlated with the traits as follows: Neuroticism, $r=0.287$; Extraversion $r=-0.131$; Openness $r=0.030$; Agreeableness $r=-0.116$; Conscientiousness $r=-0.225$. Hence, neither academic ability nor intelligence correlated reliably with the number of inductions or the personality traits.

Personality traits are not so stable across all situations ${ }^{12}$. Hence, it should be possible to elicit a temporary trait-like attitude. In a second experiment we took advantage of this possibility to manipulate the participants' attitudes and to test the effects of this manipulation on their predilection for a certain sort of reasoning. This experiment replicated the results of the first experiment after the participants had been given a fake but convincing report about their personalities. A new sample of 99 undergraduate students from Universidad de La Laguna were paid 10 euros (\$13) to participate. First, 
they completed the NEO-PI-R. One year later, they carried out the experiment. They were assigned at random to one of three groups receiving fake feedback about their personalities signed by the professor on their personality course: the open group (high in openness and low in conscientiousness), the conscientious group (low in openness and high in conscientiousness), and the control group (high in agreeableness and low in extraversion). The descriptions of the profiles were based on terms selected from the list of familiar personality adjectives on the five factors ${ }^{13}$. The experimenter who gave them their profiles did not know that these profiles had been assigned at random. Results confirmed that those who had learned that they were open to experience and low in conscientiousness made reliably more inductions than control participants, who had received a fake report about their extraversion and agreeableness. In contrast, those who had learned that were low in openness to experience but high in conscientiousness made reliably less inductions than the control participants (3.8 for the open group, 2.7 for the control group, and 1.9 for the conscientiousness group (Jonckheere-Terpstra trend test, $\mathrm{z}=2.069, \mathrm{p}<0.02)$.

A final experiment replicated the effect when the participants were asked to remember episodes in their lives relevant to the traits of openness and conscientiousness. A sample of 147 students from the University of Malaga was randomly assigned to three groups: open, control, and conscientious. Each group carried out a recall task, then the ambiguous reasoning task, and finally a short version of the NEO-PI-R. In the recall task, they had to recall ten episodes in their lives in which they had behaved in a given way based on phrases describing the episodes adapted from Goldberg's (1999) International Personality Item Pool representation of the NEO-PI-R. To ensure that they envisaged episodes, they had to write a sentence describing each episode that they recalled, and to rate on five-point scales how difficult it was to recall the episode and how pleasant it had been. The results showed that those who were told to remember episodes in which they were open and not so conscientious made more inductions than control participants, who were told to remember episodes in which they were 
extraverted and agreeable. In contrast, those who were told to remember episodes in which they were not open to experience but high in conscientiousness made reliably less inductions than the control participants (5.9 for the open group, 5.2 for the control group, and 4.6 for the conscientious group; Jonckheere-Terpstra non-parametric trend test, $\mathrm{z}=1.964 ; \mathrm{p}<0.025)$. The mean PAU scores for 59 participants were: 6.86 for the open group, 6.77 for the conscientious group, and 7.14 for the control group (KruskalWallis, $\mathrm{z}=0.754, \mathrm{p}>0.68$ ). However, Spearman's rank correlation revealed a marginal tendency for those scoring higher on PAU to make more deductions ( $\operatorname{tau}=-0.22$; $\mathrm{p}=0.094$; two-tailed), a result consistent with previous findings.

We conclude that differences in personality, and in particular those concerning the traits of openness and conscientiousness, do affect the characteristic ways in which individuals tend to reason. Openness and a lack of conscientiousness, whether they are stable traits or experimentally induced attitudes, lead individuals to think about possibilities outside those that the premises elicit. They are more likely to think 'outside the box'. As a result, their response to an ambiguous problem is to make inductions that explain phenomena. But, their polar opposites in personality do not go beyond the possibilities for the premises, and accordingly tend to make deductions.

\section{References}

${ }^{3}$ Stanovich, K.E. Who is Rational? Studies of Individual Differences in Reasoning

(Erlbaum, Mahwah, NJ, 1999)

${ }^{4}$ Braine, M.D.S., \& O’Brien, D.P. A theory of if: A lexical entry, reasoning program, and pragmatic principles. Psychol. Rev., 98, 182-203. (1991).

${ }^{5}$ Rips, L.The Psychology of Proof (MIT Press, Cambridge, MA, 1994). 
${ }^{6}$ Johnson-Laird, P.N. How We Reason (Oxford University Press, New York, NY, 2006).

${ }^{7}$ Costa, P.T. \& McCrae, R.R. Revised NEO Personality Inventory (Psychological Assessment Resources, Odessa, FL, 1992).

${ }^{8}$ Eysenck, H.J. \& Eysenck, M. Personality and Individual Differences. (Plenum Press, London, 1984)

${ }^{9}$ McCrae, R.R. \& Costa Jr., P.T. The stability of personality: observations and evaluations. Curr Dir Psychol Sci, 3, 173-175. (1994).

${ }^{10}$ Ackerman, P. L. \& Heggestad, E. D. Intelligence, personality, and interests: evidence for overlapping traits. Psychol. Bull., 121, 219-245. (1997).

${ }^{11}$ Thurstone, L.L. \& Thurstone, TH.G. P.M.A.: Aptitudes Mentales Primarias (TEA, Madrid, 1989).

${ }^{12}$ Mischel, W. Personality and assessment (Wiley, New York, 1968).

${ }^{13}$ Saucier, G. \& Goldberg, L.R. Evidence for the Big Five in analyses of familiar English personality adjectives. Eur. J. Pers, 10, 61-77. (1996).

\section{Acknowledgements}

We thank Juan Luis Luque, Adelia de Miguel, Ivan Moreno, Inés Ocón and Isabel Orenes for their help in running and organizing the experiments. Also, David Beltran, Sam Gluksberg, Geoffrey Goodwin, 
Sangeet Khemlani, Louis Lee, Gorka Navarrete and Paula Rubio for their helpful comments. This

research was supported by grants from the University of La Laguna to A.F., from the Spanish ministry for Science and Technology to C. S.

\section{Contributions statement}

The authors contributed equally to this work. 
Figure caption:

Figure 1.

The numbers of participants in Experiment 1 who made $0-2$ inductions (i.e., 810 deductions), 3-4 inductions, and so on, up to 9-10 inductions, for all the problems. Superimposed, the means in Openness (red) and Conscientiousness (blue) with standard error. 


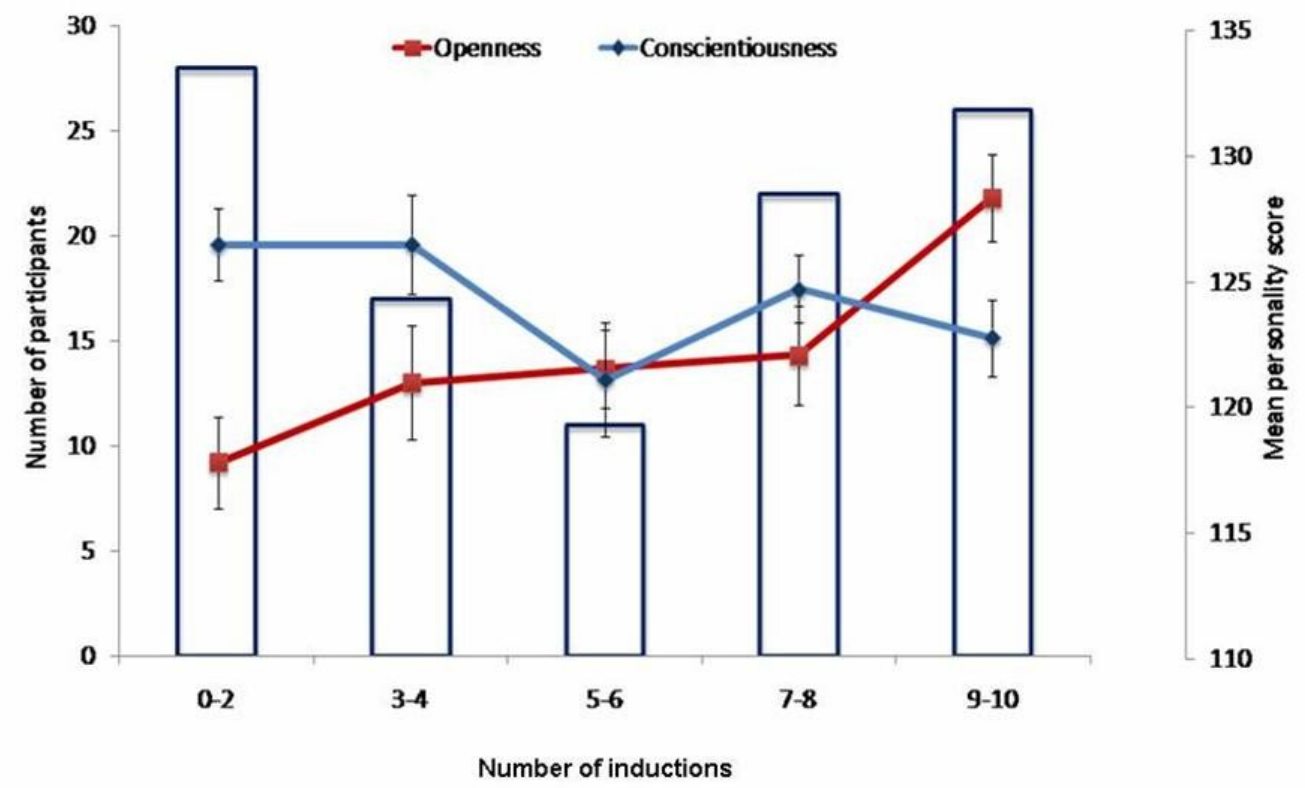

